\title{
A numerical model for the description of the lamellar and massive phase transformations in TiAl alloys
}

\author{
A. Rostamian*, A. Jacot \\ Computational Materials Laboratory, Ecole Polytechnique Fédérale de Lausanne, EPFL STI IMX LSMX MGX-317, Station 12, 1015 Lausanne, Switzerland
}

\section{A R T I C L E I N F O}

\section{Article history:}

Received 16 April 2008

Received in revised form 9 July 2008

Accepted 21 July 2008

Available online 11 September 2008

\section{Keywords:}

A. Titanium aluminides, based on TiAl

B. Phase transformations

B. Precipitates

E. Simulations

C. Heat treatment

\begin{abstract}
A B S T R A C T
A phenomenological modelling approach has been developed to describe the massive transformation and the formation of lamellar microstructures during cooling in binary $\gamma$ titanium aluminides. The modelling approach is based on a combination of nucleation and growth laws which take into account the specific mechanisms of each phase transformation. Nucleation of massive and lamellar $\gamma$ is described with classical nucleation theory, accounting for the fact that nuclei are formed predominantly at $\alpha /$ $\alpha$ grain boundaries. Growth of the massive $\gamma$ grains is based on theory for interface-controlled reactions. A modified Zener model is used to calculate the thickening rate of the $\gamma$ lamellar precipitates. The model incorporates the effect of particle impingement and coverage of the nucleation sites by the growing phase. The driving pressures of the phase transformations are obtained from Thermo-Calc based on the actual temperature and matrix composition. CCT diagrams and lamellar spacings calculated with the model are in good agreement with experimental data obtained from dedicated heat treatment experiments and from the literature. The model permitted investigating the influence of cooling rate, alloy chemistry and average $\alpha$ grain size upon the amount of massive $\gamma$ and the average thickness and spacing of the lamellae. In particular it indicates that the Al depletion of the $\alpha$ phase during lamellar precipitation seems to play an important role in the suppression of the massive transformation at moderate cooling rate and in the large lamellar spacings observed at low cooling rate.
\end{abstract}

(c) 2008 Elsevier Ltd. All rights reserved.

\section{Introduction}

Gamma titanium aluminide $(\gamma-\mathrm{TiAl})$ based alloys are very interesting materials for structural applications at elevated temperatures owing to the combination of high specific strength, good oxidation, creep and fatigue resistance. These excellent properties have made $\gamma$-TiAl alloys good candidates for replacing nickel based alloys in some gas turbine components. However, their relatively poor ductility and fracture toughness remain important obstacles for their practical application.

The ductility of $\gamma$-TiAl alloys, as well as the yield stress, creep and fracture resistance, can be considerably improved by a careful control of microstructure formation [1-3]. The microstructure of $\gamma$ TiAl alloys is essentially composed of two phases: the $\mathrm{L} 1_{0}$ ordered $\gamma$ phase and the $\alpha_{2}$ phase which is an ordered form $\left(\mathrm{DO}_{19}\right)$ of the hcp $\alpha$ phase stable at high temperature. Depending on the alloy composition and processing conditions, different types of microstructure can be obtained. Low and moderate cooling rates lead to the precipitation of $\gamma$ as parallel plates within the $\alpha$ matrix followed by the $\alpha \rightarrow \alpha_{2}$ ordering reaction. As a result of this

\footnotetext{
* Corresponding author.

E-mail address: amin.rostamian@epfl.ch (A. Rostamian).
}

transformation, the characteristic lamellar structure of TiAl is produced. Rapid cooling leads to a massive transformation from $\alpha$ to $\gamma$. Other morphologies such as feathery or Widmanstätten $\gamma$ have also been reported for intermediate conditions [4-6].

Although massive $\gamma$ microstructures do not have currently engineering applications, it has been shown that they can be used as a precursor to produce refined microstructures by subsequent tempering in the $\alpha+\gamma$ phase field [7,8]. These microstructures, which are composed of finely dispersed $\alpha_{2}$ plates with 4 variants, exhibit better mechanical properties than parallel lamellae [7]. Due to the competition with lamellar precipitation, the massive microstructure forms only at relatively high cooling rates. The critical cooling rate for the transition from lamellar to massive microstructures can be a crucial parameter for the heat treatment of large pieces where massive $\gamma$ may be difficult to obtain in the core of the component. The critical cooling rate can be lowered by the addition of heavy alloying elements such as $\mathrm{Nb}$ and $\mathrm{Ta}[9,10]$.

So far only few mathematical models have been proposed for the description of phase transformations in $\gamma$-TiAl. Analytical expressions for nucleation and growth rates of the massive grains [11-13] have been established. However, these expressions are not sufficient to calculate the overall kinetics of the phase transformation, which requires accounting for additional aspects such as grain impingement and the decrease of nucleation rates due to site 
coverage. Phase field models, which also account for the effect of stresses, have been proposed for the description of the lamellar transformation $[14,15]$. Although very innovative, such an approach is at present not applicable to complex alloys and large components for computational time reasons. Also, it does not address nucleation kinetics and possible competition with the massive transformation.

The objective of this contribution is to propose a phenomenological modelling approach for the description of the formation of lamellar and massive microstructures in TiAl during cooling. Two distinct models, for lamellar and massive microstructures, have been developed and coupled in order to describe the competition between these two microstructures. The coupled model permits investigating the influence of alloy chemistry, cooling rate and $\alpha$ grain size upon microstructural quantities such as the proportion of lamellar and massive microstructures, the size and number density of the massive grains, the lamellar spacing and phase concentrations. The model can be used to calculate CCT or TTT diagrams of TiAl alloys as a function of the composition and $\alpha$ grain size. It can be implemented into heat flow solvers for the calculation of microstructure maps in heat treated components.

\section{Background}

The main physical mechanisms associated with the lamellar and massive transformations are shortly reviewed here as they will be used to formulate the assumptions of the model. It has been established for some time that the $\alpha_{2}+\gamma$ lamellar structure is the result of a precipitation and ordering reactions rather than a eutectoid transformation [16]. Nucleation of $\gamma$ precipitates in the hcp $\alpha$ matrix is assisted by stacking faults obtained by the dissociation of a dislocation into Shockley partials and normally occurs on grain boundaries [17]. After nucleation, the precipitates, which exhibit a Blackburn relationship with the matrix [17], expand along a preferential crystallographic orientation at large velocity, whereas they thicken at a much lower rate due to the partially coherent interface. Both lateral and longitudinal extensions are assisted by a "terrace-ledge-kink" mechanism [17]. It has been shown through detailed analyses by atom probe field ion microscopy that partitioning occurs in the very early stage of the lamellar transformation [18]. These studies indicate that the lamellar transformation is a diffusion controlled phase transformation and that both the lateral and longitudinal growth rates are limited by the diffusion of alloying elements even in the very preliminary stages. Since the transformation from the hcp structure to $\mathrm{L} 1_{0}$ can be performed by the movement of partial dislocations on the interface, the transformation can be assisted by a shearing process. Stress is also believed to play a significant role in the fact that lamellae usually grow in the form of colonies [14].

The $\alpha \rightarrow \gamma$ massive transformation in TiAl has also received considerable attention, in particular regarding the aspects related to nucleation. Although bulk nucleation has been reported [9,19], it is generally accepted that nucleation of massive $\gamma$ occurs predominantly at grain boundaries. Gamma nuclei generally exhibit a Blackburn orientation relationship with one of the adjacent $\alpha$ grains while they grow at the expense of the other grain by the movement of highly mobile incoherent interfaces [20-22]. It has been suggested also that lamellae nucleated on grain boundaries serve as a precursor of massive $\gamma$ which would develop from the incoherent interface of the lamella in the adjacent grain where it has no orientation relationship [20]. Massive grains nucleated on grain boundaries grow with a hemispherical morphology owing to the immobile coherent interface with one of the adjacent $\alpha$ grains $[21,22]$. Massive growth occurs at a high velocity which is controlled by the mobility of interface and involves frequent twinning events [22]. As growth proceeds, the grain boundaries are progressively covered by massive grains and the nucleation rate diminishes.

It has been observed that the $\alpha$ grain size has an important effect on the overall kinetics of the lamellar transformation and the critical cooling rate of the massive to lamellar transition [23]. This is explained by the fact that $\gamma$ lamellae preferentially nucleate on grain boundaries due to lattice defects and possible segregation of alloying elements. A smaller grain size results therefore in higher nucleation rates and consequently faster kinetics of the lamellar transformation. It has also been reported in Ref. [23] that a smaller $\alpha$ grain size leads to a higher critical cooling rate for the formation of massive $\gamma$.

\section{Description of the model}

The lamellar and massive transformations are described with two distinct models, which are first presented separately before detailing the method used to couple them. Both the massive and the lamellar models are aimed at predicting the kinetics of a phase transformation based on the initial composition, $\alpha$ grain size and cooling path. The aspects associated with particle impingement and competition of the coexisting phases for space or nucleation sites are addressed in Section 3.3 dedicated to coupling.

\subsection{Massive model}

\subsubsection{Nucleation of massive $\gamma$}

Based on the reported observations [12,22], massive $\gamma$ grains are assumed to nucleate heterogeneously at $\alpha / \alpha$ grain boundaries. The volumetric nucleation rate is calculated with the following expression coming from classical nucleation theory:

$J_{\gamma_{\mathrm{M}}}^{V}=J_{\gamma_{\mathrm{M}}}^{S 0} S_{\mathrm{V}} S_{\alpha} \exp \left(-\frac{\Delta G_{\gamma_{\mathrm{M}}}^{\text {nucl }}\left(X_{\alpha}, T\right)}{k T}\right) \exp \left(\frac{-Q_{\mathrm{at}}}{k T}\right)$

where $J_{\gamma_{M}}^{S_{M}}$ is a prefactor expressing the number of potential nucleation sites per unit area of grain boundaries and $Q_{a t}$ is the activation energy for the atomic mobility. The activation energy for the formation of a stable atom cluster, $\Delta G_{\gamma_{M}}^{\text {nucl }}$, is given by:

$\Delta G_{\gamma M}^{\mathrm{nucl}}\left(X_{\alpha}, T\right)=f_{\theta}^{\gamma M} \frac{16 \pi \sigma_{\alpha \gamma}^{3}}{3\left(\Delta G_{\mathrm{V}}^{\alpha \rightarrow \gamma M}\left(X_{\alpha}, T\right)\right)^{2}}$

where $f_{\theta}^{\gamma_{\mathrm{M}}}$ is the wetting factor for heterogeneous nucleation of massive $\gamma, \sigma_{\alpha \gamma}$ is the interfacial energy and $\Delta G_{\mathrm{V}}^{\alpha \rightarrow \gamma_{\mathrm{M}}}\left(X_{\alpha}, T\right)$ is the volumetric driving pressure illustrated in Fig. 1. The latter is obtained from a CALPHAD approach (Thermo-Calc [24]) based on the actual atomic fraction of $\mathrm{Al}$ of the parent $\alpha$ phase, $X_{\alpha}$, and temperature.

The factor $S_{V}$ in Eq. (1) represents the surface density of grain boundaries which scales as the inverse of the average grain radius, $\bar{R}_{\text {grain }}$. In the calculations $S_{\mathrm{V}}$ will be taken equal to $3 / \bar{R}_{\text {grain }}$ which corresponds to the assumption of a spherical grain shape. The volumetric nucleation rate, $J_{\gamma_{\mathrm{M}}}^{\mathrm{V}}$, depends therefore on the $\alpha$ grain size, as it is expected for this phase transformation.

The symbol $s_{\alpha}$ denotes a weighting factor which accounts for the surface fraction of grain boundary that is still available for nucleation. It expresses the coverage of potential nucleation sites by the already formed microstructures. The method used to calculate $s_{\alpha}$ will be described in Section 3.3.

\subsubsection{Growth of massive $\gamma$}

The massive $\gamma$ grains are assumed to grow as hemispheres at a growth rate given by the Burke-Turnbull expression for interfacecontrolled reactions [25]: 


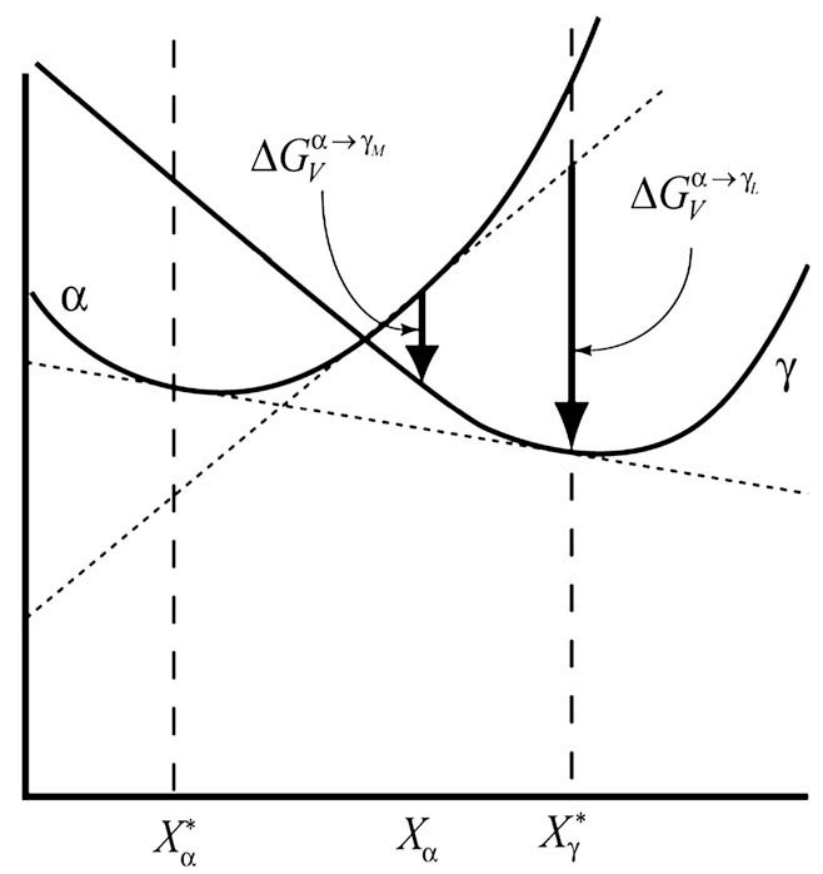

Fig. 1. Definition of the driving pressures for the $\alpha \rightarrow \gamma$ massive transformation, $\Delta G_{\mathrm{V}}^{\alpha \rightarrow \gamma_{\mathrm{M}}}$, and for the nucleation of $\gamma$ lamellae, $\Delta G_{\mathrm{V}}^{\alpha \rightarrow \gamma_{\mathrm{L}}}$, for a given temperature and $\alpha$ composition, $X_{\alpha}$.

$v_{\gamma_{\mathrm{M}}}=M_{0} \frac{-V_{\mathrm{m}} \Delta G_{\mathrm{V}}^{\alpha \rightarrow \gamma_{\mathrm{M}}}\left(X_{\alpha}, T\right)}{R T} \exp \left(\frac{-Q_{\alpha / \gamma}}{k T}\right)$

where $M_{0}$ is a mobility parameter, $Q_{\alpha / \gamma}$ is the activation energy for atomic transfer through the interface and $V_{\mathrm{m}}$ is the molar volume. As for nucleation, the driving pressure of the massive phase transformation depends on the temperature and composition of the parent $\alpha$ phase.

The volume fraction of massive $\gamma$ is calculated with the method described by Hunt [26] in order to account for continuous nucleation without having to track the entire grain size distribution. The amount of massive $\gamma$ formed during a time interval $\Delta t$ is calculated based on the first, second and third momentum of the grain size distribution as follows:

$n^{\prime}=n+J_{\gamma_{\mathrm{M}}}^{\mathrm{V}} \Delta t$

$\Delta R=v_{\gamma_{\mathrm{M}}} \Delta t$

$n \bar{R}^{\prime}=n \bar{R}+n^{\prime} \Delta R$

$n \bar{R}^{2^{\prime}}=n \bar{R}^{2}+2 n \bar{R}^{\prime} \Delta R$

$n \bar{R}^{3^{\prime}}=n \bar{R}^{3}+3 n \bar{R}^{2^{\prime}} \Delta R$

where the prime symbol stands for updated quantities after the time increment, $n$ is the volumetric density of massive $\gamma$ grains, $\Delta R$ is the radius increment of the grains and $n \bar{R}, n \bar{R}^{2}, n \bar{R}^{3}$ are the first, second and third momentum of the grain radius distribution, respectively.

As massive grains grow as hemispheres the increment of the extended volume fraction of the massive phase is given by:

$\Delta \phi_{\gamma_{\mathrm{M}}}=\frac{2}{3} \pi\left(n \bar{R}^{3^{\prime}}-n \bar{R}^{3}\right)$ which does not include the impingement of the growing phase with itself or other particles. The conversion of $\Delta \phi_{\gamma_{M}}$ to actual variations of volume fractions will be presented in the section dedicated to the coupling scheme.

Similarly, one can calculate the variation of the extended surface fraction, $\Delta \varepsilon_{\gamma_{M}}$, which corresponds to the proportion of grain boundaries occupied by massive grains if particle impingement is neglected. Considering that massive grains grow as discs on the grain boundary where they nucleated, one obtains:

$\Delta \varepsilon_{\gamma_{\mathrm{M}}}=\frac{\pi}{S_{\mathrm{V}}}\left(n \bar{R}^{2^{\prime}}-n \bar{R}^{2}\right)$

This quantity is used to account for the extinction of nucleation sites due to grain boundary coverage by the growing phase.

The $\alpha \rightarrow \alpha_{2}$ ordering transformation is not directly modelled in this work. However, it is considered to be substantially faster than the massive transformation. In order to incorporate the competition between the massive transformation and the ordering transformation, massive growth is stopped when the $T_{0}$ temperature of the $\alpha \rightarrow \alpha_{2}$ ordering transformation is reached.

\subsection{Lamellar model}

\subsubsection{Nucleation of $\gamma$ lamellae}

The model describing the lamellar microstructure is based on a similar approach as the massive model. The nucleation rate is calculated with the following expression:

$J_{\gamma_{\mathrm{L}}}^{l}=J_{\gamma_{\mathrm{L}}}^{0} s_{\alpha} \exp \left(-\frac{\Delta G_{\gamma_{\mathrm{L}}}^{\mathrm{nucl}}\left(X_{\alpha}, T\right)}{k T}\right) \exp \left(\frac{-Q_{\mathrm{at}}}{k T}\right)$

with

$\Delta G_{\gamma L}^{\mathrm{nucl}}\left(X_{\alpha}, T\right)=f_{\theta}^{\gamma M} \frac{16 \pi \sigma_{\alpha \gamma}^{3}}{3\left(\Delta G_{\mathrm{V}}^{\alpha \rightarrow \gamma L}\left(X_{\alpha}, T\right)\right)^{2}}$

where $J_{\gamma_{L}}^{0}$ is a prefactor expressing the lineal density of potential nucleation sites and $s^{\alpha}$ is the surface fraction of grain boundaries uncovered by transformation products. The driving pressure of the precipitation reaction, $\Delta G_{\mathrm{V}}^{\alpha \rightarrow \gamma_{\mathrm{L}}}$, is obtained dynamically from the thermodynamic database as a function of the temperature and matrix composition. This quantity is illustrated in Fig. 1.

The lineal nucleation rate given by Eq. (11) allows us to calculate the number of lamellae per unit length, which is also the inverse of the lamellar spacing, $1 / \lambda$, and update it at each time increment:

$\frac{1}{\lambda^{\prime}}=\frac{1}{\lambda}+J_{\gamma_{\mathrm{L}}}^{l} \Delta t$

\subsubsection{Lamellar growth}

Lamellae are assumed to grow in the form of colonies developing from the boundaries towards the interior of the grain. A classical expression for the longitudinal growth rate of plate-like precipitates is used to estimate the expansion rate of the colony. Neglecting the capillary effects the growth rate at lamella edges is given by:

$v_{\gamma_{\mathrm{L}}}^{\text {lengthening }}=\frac{\tilde{D}_{\alpha}\left(X_{0}-X_{\alpha}^{*}\right)}{b\left(X_{\gamma}^{*}-X_{\alpha}^{*}\right)} \frac{1}{r}$

where $r$ is the edge radius of the plates, $b$ is a geometrical factor, $X_{0}$ is the nominal atomic fraction of $\mathrm{Al}$, and $X_{\alpha}^{*}$ and $X_{\gamma}^{*}$ are the 
equilibrium atomic fraction of $\mathrm{Al}$ in $\alpha$ and $\gamma$ at the current temperature, respectively.

The interdiffusion coefficient of $\mathrm{Al}$ in $\alpha, \tilde{D}_{\alpha}$, is obtained with the following expression:

$\tilde{D}_{\alpha}=X_{\alpha}^{*} D_{0}^{\mathrm{Al}} \exp \left(-Q^{\mathrm{Al}} / R T\right)+\left(1-X_{\alpha}^{*}\right) D_{0}^{\mathrm{Ti}} \exp \left(-Q^{\mathrm{Ti}} / R T\right)$

where $D_{0}^{\mathrm{Al}}, D_{0}^{\mathrm{Ti}}, Q^{\mathrm{Al}}$ and $Q^{\mathrm{Ti}}$ are parameters of thermally activated expressions for the self-diffusion coefficients.

It is assumed that the lamellar colonies grow from the grain boundaries towards the interior of the grain and have a uniform thickness, $l_{\text {colony }}$, which is calculated by integration of Eq. (14). The colony thickness is given by the maximum length of the lamellae, i.e. the length of the first nucleated lamella. It should be noticed that the concept of colony introduced in this model differs somewhat from the usual definition of lamellar colonies. In the model, the lamellar colonies refer to the regions in the $\alpha$ grain that are potentially affected by the growth of $\gamma$ lamellae growing from the grain boundaries. This concept is essentially used to define the proportion of the $\alpha$ matrix that is chemically affected by the precipitation of $\gamma$.

Under these assumptions, the extended volume fraction of lamellar colonies is given by:

$\phi=\frac{\bar{R}_{\text {grain }}^{3}-\left(\bar{R}_{\text {grain }}-l_{\text {colony }}\right)^{3}}{\bar{R}_{\text {grain }}^{3}}$

The possible impingement of lamellar colonies with other microstructures, which is not included in this expression, will be presented in Section 3.3.

The thickening of the lamellae is considered as a one-dimensional diffusion problem which can be described with a Zener model [27] as illustrated in Fig. 2. The following coupled equations are solved:

$w\left(X_{\gamma}^{*}-\bar{X}_{\alpha+\gamma_{\mathrm{L}}}^{\mathrm{col}}\right)=\frac{\delta}{2}\left(\bar{X}_{\alpha+\gamma_{\mathrm{L}}}^{\mathrm{col}}-X_{\alpha}^{*}\right)$

$v_{\gamma_{\mathrm{L}}}^{\text {thickening }}\left(X_{\gamma}^{*}-X_{\alpha}^{*}\right)=\frac{h_{\tilde{D}_{\alpha}}}{l} \frac{\left(\bar{X}_{\alpha+\gamma_{\mathrm{L}}}^{\mathrm{col}}-X_{\alpha}^{*}\right)}{\delta}$

where $\delta$ is the depth of the boundary diffusion layer in the $\alpha$ matrix, $w$ is the thickness of the $\gamma$ lamellae, $v_{\gamma_{L}}^{\text {thickening }}$ is the interface velocity and $\bar{X}_{\alpha+\gamma_{L}}^{\text {col }}$ is the average atomic fraction of $\mathrm{Al}$ in the lamellar colony.

To account for the fact that $\gamma$ lamellae grow by a ledge mechanism, a geometrical factor $h / l$ is introduced into the interface solute balance (Eq. (18)). This factor, which represents the height over length ratio of the ledges, was estimated from TEM observations $[28,29]$ and set to 0.02 for all calculations.
When $w+\delta$ reaches half of the lamellar spacing, $\lambda / 2$, the diffusion layers of two adjacent lamellae start to interact. Growth is then described with another set of equations:

$w\left(X_{\gamma}^{*}-\bar{X}_{\alpha+\gamma_{\mathrm{L}}}^{\mathrm{col}}\right)=\frac{\lambda / 2-w}{2}\left(2 \bar{X}_{\alpha+\gamma_{\mathrm{L}}}^{\mathrm{col}}-X_{\alpha}^{*}-X_{\mathrm{b}}\right)$

$v_{\gamma_{\mathrm{L}}}^{\text {thickening }}\left(X_{\gamma}^{*}-X_{\alpha}^{*}\right)=\frac{h}{l} D_{\alpha} \frac{X_{\mathrm{b}}-X_{\alpha}^{*}}{\lambda / 2-w}$

where $X_{\mathrm{b}}$ is the $\mathrm{Al}$ atomic fraction in the centre of the $\alpha$ region (see Fig. 2b).

The variation of the extended volume fraction of lamellar $\gamma$ is given by:

$\Delta \phi_{\gamma_{\mathrm{L}}}^{\mathrm{col}}=2 g^{\mathrm{col}} v_{\gamma_{\mathrm{L}}}^{\text {thickening }} \Delta t / \lambda$

where $g^{\mathrm{col}}$ is the volume fraction of lamellar colonies.

\subsection{Competition between the lamellar and massive microstructures}

The principles used to describe microstructure competition are schematically shown in Fig. 3. As cooling proceeds the parent $\alpha$ phase transforms progressively into lamellar colonies and/or massive $\gamma$ grains. Besides $\gamma$ precipitates the lamellar colonies include also some $\alpha$ phase which is no longer at nominal concentration since $\gamma$ precipitation depletes the matrix in Al. As $\gamma$ lamellae thicken much slower than they lengthen, the colony may contain a very large amount of $\alpha$ phase. This is particularly pronounced with the definition of colonies used in this approach, where all the regions that can possibly be reached by $\gamma$ lamellae growing from the boundaries are considered to be part of the colony. With such a definition, massive $\gamma$ can well nucleate and grow inside the so-called colony as $\alpha$ may be only slightly depleted in Al. The massive model is therefore applied to two distinct regions which correspond to the $\alpha$ phase outside and inside the colonies.

The microstructure is assumed to be composed of five constituents: lamellar $\gamma$, massive $\gamma$ outside the colonies, massive $\gamma$ inside the colonies, remaining $\alpha$ outside the colonies and remaining $\alpha$ inside the colonies. In the equations presented hereafter the volume fractions of the different constituents listed above are denoted with the following notation: $g_{\gamma_{L}}^{\text {col }}, g_{\gamma_{M}}^{\text {out }}, g_{\gamma_{M}}^{\text {col }}, g_{\alpha}^{\text {out }}, g_{\alpha}^{\text {col }}$ where the exponents "out" and "col" stand for outside and inside the colonies, respectively. Similarly, a fraction of occupied surface on grain boundaries is defined for each constituent: $s_{\gamma_{L}}^{\text {col }}, s_{\gamma_{M}}^{\text {out }}, s_{\gamma_{M}}^{\text {col }}, s_{\alpha}^{\text {out }}$ and $s_{\alpha}^{\text {col }}$. The following equations are always satisfied:

$g_{\gamma_{L}}^{\text {col }}+g_{\gamma_{M}}^{\text {out }}+g_{\gamma_{M}}^{\text {col }}+g_{\alpha}^{\text {out }}+g_{\alpha}^{\text {col }}=1$ $\mathbf{a}$

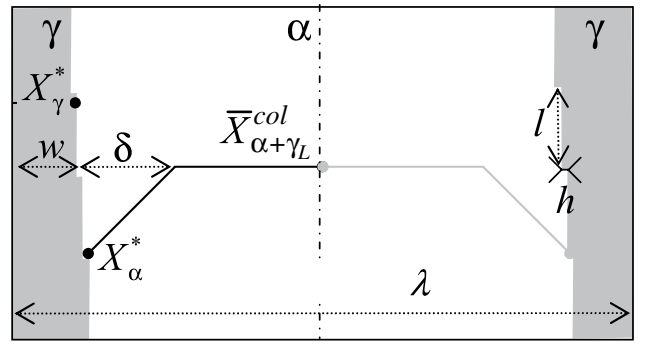

b

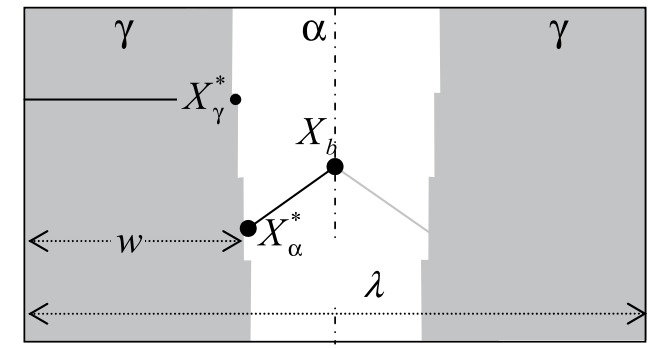

Fig. 2. Schematic Al concentration profile during the lateral growth of $\gamma$ lamellae before (a) and after (b) impingement of the diffusion boundary layers. 


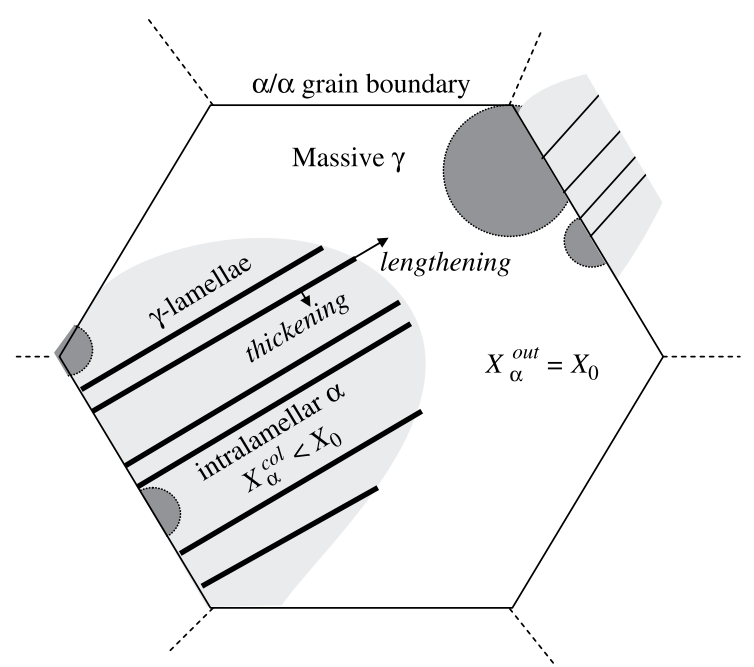

Fig. 3. Schematic representation of the modelling approach used to address the competition between the microstructure constituents.

$s_{\gamma_{\mathrm{L}}}^{\mathrm{col}}+s_{\gamma_{\mathrm{M}}}^{\text {out }}+s_{\gamma_{\mathrm{M}}}^{\mathrm{col}}+s_{\alpha}^{\text {out }}+s_{\alpha}^{\mathrm{col}}=1$

The competition between the different microstructures is described with an incremental procedure over time. The operations performed during a time interval can be decomposed into 4 steps: (1) nucleation of $\gamma$ lamellae and expansion of the colonies, (2) thickening of $\gamma$ lamellae in the colonies, (3) nucleation and growth of massive $\gamma$ outside the colonies, (4) nucleation and growth of massive $\gamma$ inside the colonies. The average concentration of $\alpha$ inside the colonies, $X_{\alpha}^{\text {col }}$, and the average concentration of lamellar $\gamma$ and $\alpha$ in the colonies $\bar{X}_{\alpha+\gamma,}^{\text {col }}$ are updated after each step as these quantities are used to calculate the nucleation and growth rates. The variation of volume fractions corresponding to steps 1-4 within a given time interval are denoted with an additional index: \#1, \#2, \#3 and \#4.

During each step the extended surface and volume fractions obtained from the massive or lamellar models are converted into actual volume and surface fractions taking into account the presence of the other phases. The method relies on statistical rules which are essentially based of the concept of particle impingement developed by Avrami [30]. More precisely, the volume fraction increments are obtained by multiplying the corresponding extended volume increment by an impingement factor, the latter being defined as the probability for a moving interface to be free of any obstacle.

The sequence of operations performed during a time interval can be described as follows.

Step 1: nucleation of $\gamma$ lamellae and expansion of the colonies. The nucleation rate of the $\gamma$ lamellae is calculated with Eqs. (11) and (12), using $s_{\alpha}=s_{\alpha}^{\text {out }}+s_{\alpha}^{\text {col }}$ to account for the coverage of the nucleation sites, and a driving pressure calculated for the average concentration of $\alpha$ inside the colonies, $X_{\alpha}^{\text {col }}$. The lamellar spacing is then updated with Eq. (13). The expansion rate, $v_{\gamma_{L}}^{\text {lengthening, }}$, and the corresponding variation of extended volume fraction of the colonies, $\Delta \phi_{\# 1}^{\text {colony }}$, are calculated with Eqs. (14) and (16), respectively. As lamellar colonies can only grow at the expense of the $\alpha$ phase located outside, the impingement factor is taken as $g_{\alpha}^{\text {out }} /\left(g_{\gamma_{M}}^{\text {out }}+g_{\alpha}^{\text {out }}\right)$. This expression is based on the assumption that the only possible obstacles for the growth of the colony are massive $\gamma$ particles that are homogeneously distributed in the region outside the colony. The fact that massive $\gamma$ nucleate predominantly at grain boundaries is not considered in this approach of particle impingement. This simplification is justified by the fact that once the conditions for the massive transformation are met, massive $\gamma$ particles grow very rapidly and almost immediately stop the lamellar colonies, regardless of their spatial distribution. The volume fraction variations associated with the expansion of the lamellar colony are obtained with the following expressions:

$\Delta g_{\# 1}^{\text {col }}=-\Delta g_{\alpha, \# 1}^{\text {out }}=g_{\alpha}^{\text {out }} /\left(g_{\gamma_{M}}^{\text {out }}+g_{\alpha}^{\text {out }}\right) \Delta \phi_{\# 1}^{\text {col }}$

$\Delta g_{\gamma_{\mathrm{M}}, \# 1}^{\mathrm{col}}=\Delta g_{\gamma_{\mathrm{M}}, \# 1}^{\text {out }}=0$

$\Delta g_{\alpha, \# 1}^{\mathrm{col}}=g_{\alpha}^{\mathrm{col}} /\left(g_{\gamma_{\mathrm{L}}}^{\mathrm{col}}+g_{\alpha}^{\mathrm{col}}\right) \Delta g_{\# 1}^{\mathrm{col}}$

$\Delta g_{\gamma_{L}, \# 1}^{\mathrm{col}}=g_{\gamma_{\mathrm{L}}}^{\mathrm{col}} /\left(g_{\gamma_{\mathrm{L}}}^{\mathrm{col}}+g_{\alpha}^{\mathrm{col}}\right) \Delta g_{\# 1}^{\mathrm{col}}$

These equations are based on the assumptions that the volume fraction of massive $\gamma$ is not affected by the expansion of the colonies and that the proportions of lamellar $\gamma$ and $\alpha$ within the colonies remain constant. A similar method is used to update the surface fraction of the colony at grain boundaries assuming that the extended surface fraction of lamellar colonies is equal to its extended volume fraction.

As the average concentration of $\alpha$ inside the colony, $X_{\alpha}^{\text {col }}$, is not necessarily equal to that of the captured volume, it is corrected by an amount $\Delta X_{\alpha, \# 1}^{\mathrm{col}}$. By assuming equal densities for all microstructure constituents, $\Delta X_{\alpha, \# 1}^{\mathrm{col}}$ is obtained with the following solute balance:

$\Delta g_{\# 1}^{\mathrm{col}} X_{0}=\Delta g_{\gamma L, \# 1}^{\mathrm{col}} X_{\gamma}^{*}+\Delta g_{\alpha, \# 1}^{\mathrm{col}}\left(X_{\alpha}^{\mathrm{col}}+\Delta X_{\alpha, \# 1}^{\mathrm{col}}\right)+g_{\alpha}^{\mathrm{col}} \Delta X_{\alpha, \# 1}^{\mathrm{col}}$

The average concentration in the lamellar colonies is then recalculated:

$\bar{X}_{\alpha+\gamma}^{\mathrm{col}}=\left(g_{\gamma \perp}^{\mathrm{col}}+\Delta g_{\gamma \perp, \# 1}^{\mathrm{col}}\right) X_{\gamma}^{*}+\left(g_{\alpha}^{\mathrm{col}}+\Delta g_{\alpha, \# 1}^{\mathrm{col}}\right)\left(X_{\alpha}^{\mathrm{col}}+\Delta X_{\alpha, \# 1}^{\mathrm{col}}\right)$

Step 2: thickening of $\gamma$ lamellae. The thickening of $\gamma$ lamellae in the colony is calculated with Eqs. (17-21). The impingement factor is taken as $g_{\alpha}^{\mathrm{col}} /\left(g_{\gamma_{\mathrm{M}}}^{\mathrm{col}}+g_{\alpha}^{\mathrm{col}}\right)$, which means that the $\gamma$ lamellae only thicken at the expense of $\alpha$ located within the colony and that impingement with other lamellae is neglected. The latter assumption is justified by the fact that the lamellae are parallel. The volume fraction variations corresponding to this step of the algorithm are given by:

$\Delta g_{\gamma_{L}, \# 2}^{\mathrm{col}}=-\Delta g_{\alpha, \# 2}^{\mathrm{col}}=g_{\alpha}^{\mathrm{col}} /\left(g_{\gamma_{\mathrm{M}}}^{\mathrm{col}}+g_{\alpha}^{\mathrm{col}}\right) \Delta \phi_{\gamma_{\mathrm{L}}, \# 2}^{\mathrm{col}}$

$\Delta g_{\gamma_{M}, \# 2}^{\text {col }}=\Delta g_{\gamma_{M}, \# 2}^{\text {out }}=\Delta g_{\alpha, \# 2}^{\text {out }}=0$

where $\Delta \phi_{\gamma_{\mathrm{L}}, \# 2}^{\mathrm{col}}$ is obtained from Eq. (21).

A similar method is used to update the surface fraction of lamellar $\gamma$ at grain boundaries assuming that the extended surface fraction of $\gamma$ lamellae varies in the same way as the extended volume fraction.

The variation of the average concentration in $\alpha$ within the colony due to thickening of the $\gamma$ plates, $\Delta X_{\alpha, \# 2}^{\text {col }}$, is calculated by performing a solute balance over the $\alpha$ and $\gamma$ lamellae, assuming that the average concentration in the lamellar colony, $\bar{X}_{\alpha+\gamma_{L}}^{\text {col }}$, is left unchanged.

$$
\begin{aligned}
& \left(g_{\gamma_{\mathrm{L}}}^{\mathrm{col}}+\Delta g_{\gamma_{\mathrm{L}}, \# 2}^{\mathrm{col}}\right) X_{\gamma}^{*}+\left(g_{\alpha}^{\mathrm{col}}+\Delta g_{\alpha, \# 2}^{\mathrm{col}}\right)\left(X_{\alpha}^{\mathrm{col}}+\Delta X_{\alpha, \# 2}^{\mathrm{col}}\right) \\
& =\left(g_{\gamma_{\mathrm{L}}}^{\mathrm{col}}+g_{\alpha}^{\mathrm{col}}\right) \bar{X}_{\alpha+\gamma_{\mathrm{L}}}^{\mathrm{col}}
\end{aligned}
$$


Step 3: nucleation and growth of massive $\gamma$ outside the colonies. The nucleation rate and the variation of extended volume and surface fractions of massive grains growing outside the colonies, $\Delta \phi_{\gamma_{\mathrm{M}}, \# 3}^{\text {out }}$ and $\Delta \varepsilon_{\gamma_{\mathrm{M}}, \# 3}^{\text {out }}$, are calculated with Eqs. (9) and (10) using $s_{\alpha}=s_{\alpha}^{\text {out }}$ to account for grain boundary coverage in Eq. (1) and the nominal composition $X_{\alpha}^{\text {out }}=X_{0}$ for the calculation of the driving force. The variations of the volume fractions are calculated with the assumption of a random spatial distribution of the transformed regions:

$\Delta g_{\gamma_{M}, \# 3}^{\text {out }}=-\Delta g_{\alpha, \# 3}^{\text {out }}=g_{\alpha}^{\text {out }} \Delta \phi_{\gamma_{\mathrm{M}}, \# 3}^{\text {out }}$

$\Delta g_{\gamma_{\mathrm{L}}, \# 3}^{\mathrm{col}}=\Delta g_{\alpha, \# 3}^{\mathrm{col}}=\Delta g_{\gamma_{\mathrm{M}}, \# 3}^{\mathrm{col}}=0$

and similarly for the surface fraction:

$\Delta s_{\gamma_{M}, \# 3}^{\text {out }}=-\Delta s_{\alpha, \# 3}^{\text {out }}=s_{\alpha}^{\text {out }} \Delta \varepsilon_{\gamma_{M}, \# 3}^{\text {out }}$

$\Delta s_{\gamma_{L}, \# 3}^{\mathrm{col}}=\Delta s_{\alpha, \# 3}^{\mathrm{col}}=\Delta s_{\gamma_{\mathrm{M}}, \# 3}^{\mathrm{col}}=0$

Step 4: nucleation and growth of massive $\gamma$ inside the colonies. The same procedure as in step (3) is used to calculate the variation of the volume and surface fractions of massive $\gamma$ growing inside the colonies, $\Delta g_{\gamma_{M}, \# 4}^{\text {col }}$ and $\Delta s_{\gamma_{M}, \# 4}^{\text {col }}$, except for the coverage factor of Eq. (1) which is taken as $s_{\alpha}=s_{\alpha}^{\text {col }}$ and the driving force which is calculated based on the actual composition of $\alpha$ in the colony, $X_{\alpha}^{\text {col }}$. Since there is no solute partitioning taking place during massive growth, newly formed massive $\gamma$ always inherits the current composition of the $\alpha$ phase, $X_{\alpha}^{\text {col }}$. Solute redistribution within massive $\gamma$ formed at different compositions is not considered due to the fast kinetics of this reaction.

\section{Experimental}

Heat treatment experiments have been carried out in order to measure the transformation kinetics and produce samples for microstructure analysis. The experiments consisted of heating up TiAl samples in the $\alpha$ phase field and cooling down with various rates. The objective is to measure the starting and ending temperatures of the phase transformations and analyze the microstructure in terms of volume fraction of constituents and lamellar spacing. The experiments were conducted inside a differential scanning calorimeter (DSC) chamber or in an infrared furnace. Both instruments work in a protective atmosphere and have the capability to register the temperature history of the sample during the thermal cycle. The experiments were conducted on a $\mathrm{Ti}-48$ at\% $\mathrm{Al}$ alloy produced by powder metallurgy. This alloy was characterized in detail by Dudzinski et al. in terms of chemistry and production procedure [31].

The samples used for the heat treatment experiments carried out in the DSC apparatus were small discs of $40 \mathrm{mg}$ machined out from cylindrical bars by electric discharged machining (EDM). The samples were placed inside a $300 \mathrm{mg}$ alumina crucible and an empty crucible was employed as a reference. A $5 \mathrm{ml} / \mathrm{min}$ flow of Argon was used as a protective atmosphere.

The thermal cycle consisted of ramping the temperature up to $1385^{\circ} \mathrm{C}$ at a rate of $0.33^{\circ} \mathrm{C} / \mathrm{s}$, holding for $300 \mathrm{~s}$ and cooling down at a controlled rate of $0.33{ }^{\circ} \mathrm{C} / \mathrm{s}$ or, alternatively, letting the system cool down naturally, which corresponds to a decreasing cooling rate from $1.6^{\circ} \mathrm{C} / \mathrm{s}$ at $1350{ }^{\circ} \mathrm{C}$ to $1.33^{\circ} \mathrm{C} / \mathrm{s}$ at $1100{ }^{\circ} \mathrm{C}$ and $0.66^{\circ} \mathrm{C} / \mathrm{s}$ at $700^{\circ} \mathrm{C}$. The starting and ending temperatures were determined from the dips that could be clearly identified in the curves of the cooling rate reported as a function of time. The results were then reported on a continuous cooling transformation (CCT) diagram.
As the cooling rate in the DSC apparatus (NETZSCH 404C) is limited to approximately $1.7^{\circ} \mathrm{C} / \mathrm{s}$, additional experiments were carried out in an infrared furnace (ULVAC-RIKO VST-E48) which allows for fast heating and cooling. The set up was modified in order to perform heat treatment under vacuum. The temperature was measured continuously by a B type thermocouple which was spot-welded at the surface of a $0.025 \mathrm{~mm}$ thick $99.9 \% \mathrm{Ta}$ foil wrapped around the sample to prevent surface reactions.

The samples for the optical microscopy were polished with colloidal silica and etched with a $25 \mathrm{ml} \mathrm{HNO}_{3}+2 \mathrm{ml} \mathrm{HF}+50 \mathrm{ml}$ glycerine $+25 \mathrm{ml} \mathrm{H}_{2} \mathrm{O}$ solution in order to reveal the $\alpha$ and $\gamma$ phases according to the procedure described in Ref. [32]. SEM/BS observations were performed on the polished samples before etching.

The lamellar spacing was measured in SEM using the backscattered secondary electron mode. The measurements were performed by a semiautomatic method based on the extraction of the average grey level from the SEM images. The spacing was measured by counting the intersections between the different grey zones along lines drawn perpendicularly to the lamellae. The measured spacings were multiplied by a factor $\pi / 4$ to correct for the fact that the apparent spacing in a cross-section is statistically larger than the true spacing. The method permitted to assess the distribution of the lamellar spacing in each sample and extract the average value and the standard deviation.

\section{Results and discussion}

The model was applied to Ti-47.5 at\% $\mathrm{Al}$ and $\mathrm{Ti}-48$ at\% $\mathrm{Al}$. The thermodynamic data needed for the calculations, i.e. the driving pressures and the equilibrium phase compositions $X_{\alpha}^{*}$ and $X_{\gamma}^{*}$, were taken from Thermo-Calc [24] and the database SSOL4 from SGTE Solutions Database. The phase diagram corresponding to this data is shown in Fig. 4. A tabulation procedure of $X_{\alpha}^{*} \Delta G_{\mathrm{V}}^{\alpha \rightarrow \gamma L}$ and $X_{\gamma}^{*} \Delta G_{\mathrm{V}}^{\alpha \rightarrow \gamma M}$ vs. temperature and of the driving pressures, $\Delta G_{\mathrm{V}}$ and $\Delta G_{\mathrm{V}}$, vs. temperature and $X_{\alpha}$ was utilized in order to avoid repetitive calls to Thermo-Calc and the practical difficulties associated with direct coupling. All the other parameters are listed in Table 1. The diffusion coefficients were taken from Ref. [33] and the mobility parameters entering into the Burke-Turnbull expression for the rate of massive growth $\left(M_{0}\right.$ and $Q_{\alpha / \gamma}$ in Eq. (3)) were those

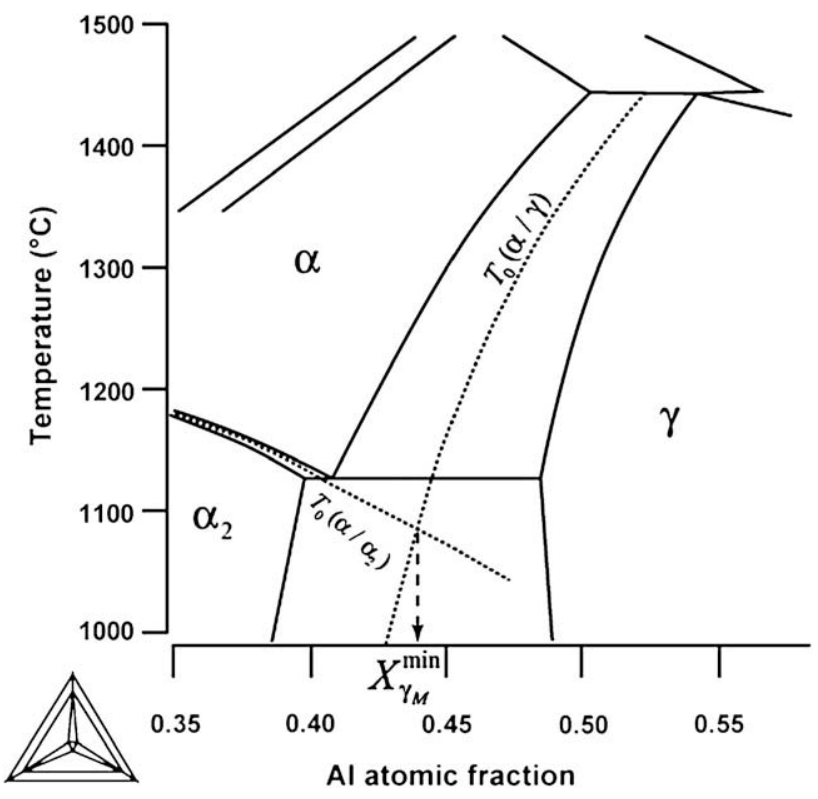

Fig. 4. Ti-Al phase diagram used for the calculations. Drawn with Thermo-Calc [24] and the database SSOL4 from SGTE Solutions Database. 
Table 1

Parameters of the calculations

\begin{tabular}{llll}
\hline Parameter & Source & Value & Unit \\
\hline$J_{\gamma_{\mathrm{M}}}^{\gamma_{\mathrm{M}}}$ & Adjusted & $1.4 \times 10^{13}$ & $\mathrm{~m}^{-2} \mathrm{~s}^{-1}$ \\
$f_{\theta}^{\gamma_{\mathrm{M}}}$ & Adjusted & 0.01 & - \\
$J_{\gamma L}^{0}$ & Adjusted & $1.2 \times 10^{9}$ & $\mathrm{~m}^{-1} \mathrm{~s}^{-1}$ \\
$f_{\theta}^{\gamma_{\mathrm{L}}}$ & Adjusted & 0.005 & - \\
$Q_{\text {at }}$ & {$[12]$} & $2.578 \times 10^{-19}$ & $\mathrm{~J}$ \\
$Q_{\alpha / \gamma}$ & {$[13]$} & $2.578 \times 10^{-19}$ & $\mathrm{~J}$ \\
$M_{0}$ & {$[13]$} & $2.302 \times 10^{4}$ & $\mathrm{~m} \mathrm{~s}^{-1}$ \\
$h / l$ & {$[28,29]$} & 0.02 & - \\
$b$ & - & 1.0 & - \\
$\sigma_{\alpha \gamma}$ & {$[12]$} & 0.35 & $\mathrm{~J} \mathrm{~m}^{-2}$ \\
$D_{0}^{\mathrm{Al}}$ & {$[33]$} & $6.65 \times 10^{-3}$ & $\mathrm{~m}^{2} \mathrm{~s}^{-1}$ \\
$Q^{\mathrm{Al}}$ & {$[33]$} & 329,000 & $\mathrm{~J} \mathrm{~mol}^{-1}$ \\
$D_{0}^{\mathrm{Ti}}$ & {$[33]$} & $1.35 \times 10^{-3}$ & $\mathrm{~m}^{2} \mathrm{~s}^{-1}$ \\
$Q^{\mathrm{Ti}}$ & {$[33]$} & 303,000 & $\mathrm{~J} \mathrm{~mol}^{-1}$ \\
\hline
\end{tabular}

established by Veeraraghavan et al. based on measurements of the starting and ending temperatures in Ti-47.5Al [13]. The four nucleation parameters of the model, $J_{\gamma_{M}}^{S 0}, J_{\gamma_{L}}^{0}, f_{\theta}^{\gamma_{M}}$ and $f_{\theta}^{\gamma_{L}}$, have been considered as adjustable parameters as they are virtually impossible to measure. These parameters were determined from the experimental data obtained in the present work for Ti-48Al and the data reported in Ref. [13] for massive transformation in Ti-47.5Al. They were adjusted in order to obtain good agreement between calculated and measured start temperatures at different cooling rates. The same set of parameters was then used for all calculations, assuming it remains valid for other $\mathrm{Al}$ contents and $\alpha$ grain sizes.

\subsection{CCT diagrams and microstructures}

The model was used to construct CCT diagrams for various compositions and grain sizes by performing successive runs at different cooling rates. Fig. 5 illustrates the CCT diagrams calculated for $\mathrm{Ti}-48$ at\% $\mathrm{Al}$ and $\mathrm{Ti}-47.5$ at\% $\mathrm{Al}$ and an average $\alpha$ grain size of $\bar{R}_{\text {grain }}=150 \mu \mathrm{m}$. The dashed and solid lines are isopleths of the volume fractions of massive and lamellar phases, respectively.

As can be seen in Fig. 5a, a fully lamellar microstructure is predicted at low and moderate cooling rates, i.e. for $\dot{T}<60^{\circ} \mathrm{C} / \mathrm{s}$. The starting temperature of the lamellar transformation, which can be defined by the isopleth $g_{\gamma_{L}}=0.1$, strongly depends on $\dot{T}$ as can be expected for phase transformations occurring by nucleation and growth. While the isopleth $g_{\gamma_{\mathrm{L}}}=0.1$ has a positive slope over the

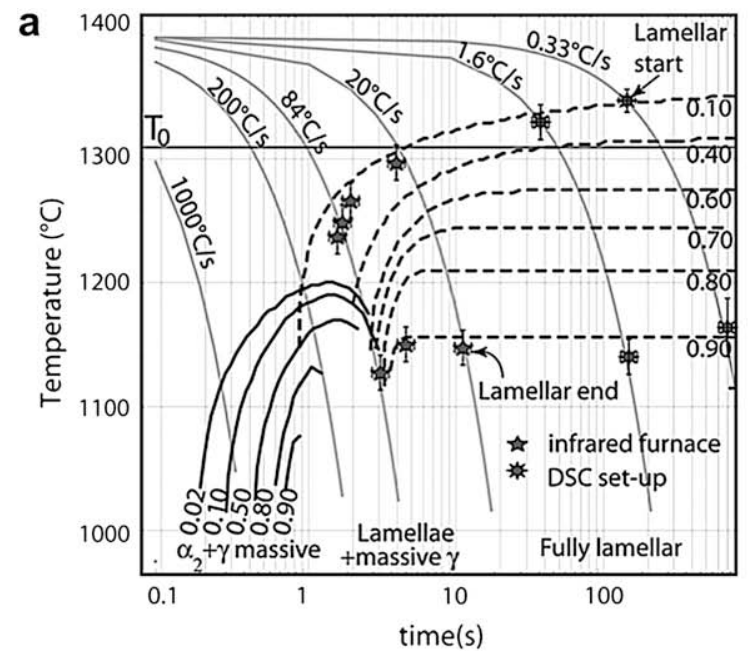

entire range of cooling rates, it can be observed that $g_{\gamma_{L}}=0.9$ is almost horizontal on the right hand side of the diagram. It reflects the fact that the fraction of lamellar $\gamma$ reaches thermodynamic equilibrium at very low $\dot{T}$.

For increasing $\dot{T}$ lamellar $\gamma$ is gradually replaced by the massive microstructure. For $\dot{T}$ comprised between 60 and $200^{\circ} \mathrm{C} / \mathrm{s}$ the lamellar and massive microstructures coexist. The critical cooling rate to obtain massive $\gamma$, which can be defined as $50 \%$ of massive microstructure after cooling, is almost the same for Ti-48Al and for Ti-47.5Al and is about $75^{\circ} \mathrm{C} / \mathrm{s}$. Both the massive and the lamellar start temperatures are substantially lower for Ti-47.5Al than for $\mathrm{Ti}-48 \mathrm{Al}$ due to the slopes of the $T_{0}$ and $T_{\alpha}$ lines in the phase diagram (see Fig. 4). One can note that the massive transformation starts at a temperature about $120^{\circ} \mathrm{C}$ below $T_{0}$, which translates the fact that high undercoolings are required to nucleate and/or grow the massive phase. At large cooling rates $\left(\dot{T}>200^{\circ} \mathrm{C} / \mathrm{s}\right)$ the formation of lamellar $\gamma$ is no longer expected. The microstructure is, however, not entirely composed of massive $\gamma$ grains as some $\alpha$ phase is retained. The amount of retained $\alpha$ increases with the cooling rate.

The start temperatures of the phase transformation determined experimentally in the DSC and infrared setups and other data from the literature [13] have also been reported in Fig. 5a and b for the sake of comparison with the first isopleths of $g_{\gamma_{L}}$ and $g_{\gamma_{M}}$. A good agreement is obtained over the entire range of cooling rates. This is naturally due to the fact that the nucleation parameters were adjusted based on the same set of experimental data. One can note, however, that good agreement is obtained over a very large range of cooling rates with only four adjustable nucleation parameters. In addition, as can be seen in Fig. 6 , the model reproduces satisfactorily the amounts of retained $\alpha$ estimated from the micrographs reported in Ref. [13] for Ti-47.5Al in the massive regime. This information was not used to determine the adjustable parameters.

Micrographs of Ti-48Al samples cooled at $1.6^{\circ} \mathrm{C} / \mathrm{s}$ (DSC) and $76{ }^{\circ} \mathrm{C} / \mathrm{s}$ (infrared furnace) are shown in Fig. 7. A fully lamellar microstructure is obtained at $1.6^{\circ} \mathrm{C} / \mathrm{s}$ whereas the presence of both massive and lamellar $\gamma$ is observed at $76{ }^{\circ} \mathrm{C} / \mathrm{s}$, which is in agreement with the calculations.

\subsection{Lamellar spacing}

The lamellar spacings calculated with the model for Ti-48Al are shown in Fig. 8. It can be seen that $\lambda$ is a strong function of $\dot{T}$ as it ranges from $0.1 \mu \mathrm{m}$ at $30^{\circ} \mathrm{C} / \mathrm{s}$ to about $6 \mu \mathrm{m}$ at slow rates. The average lamellar spacing measured in samples cooled down at 1.6,

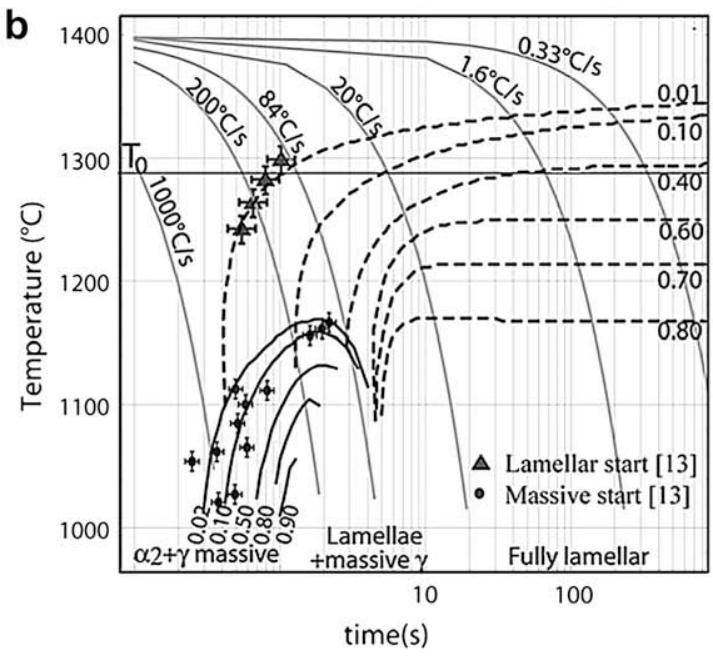

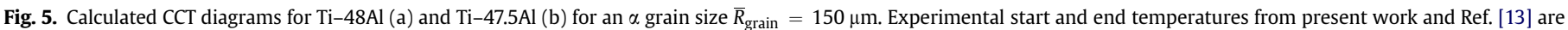
represented with symbols. 


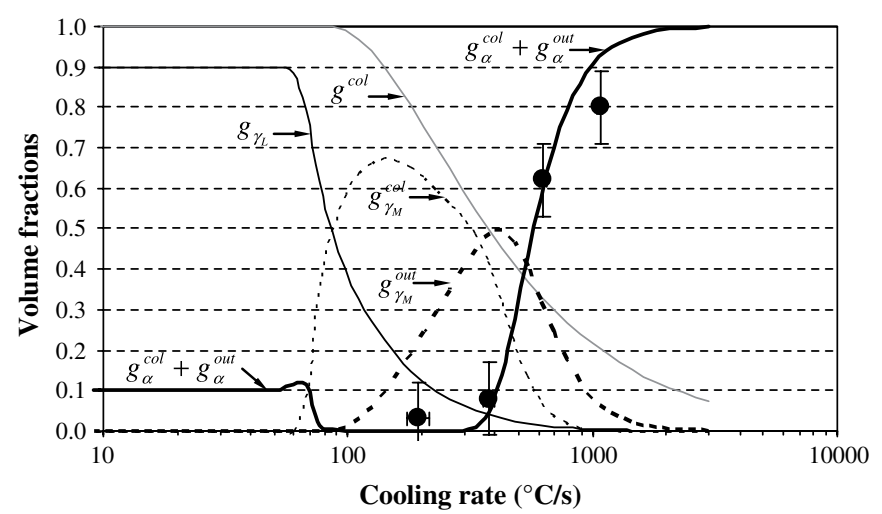

Fig. 6. Calculated volume fractions of the microstructure constituents as a function of the cooling rate in Ti-47.5Al. The experimental volume fractions of retained $\alpha$ (symbols) were estimated from the results reported in Ref. [13].

22 and $67^{\circ} \mathrm{C} / \mathrm{s}$ have been reported on the same diagram together with additional data taken from the literature for the same alloy [34]. It can be seen that simulation results are in good agreement with the measurements.

The model can be used to analyze the physical mechanisms responsible for the observed $\lambda(\dot{T})$ dependency. The lamellar spacing is essentially determined by the nucleation behaviour, as it is directly related to the density of precipitates. The growth of $\gamma$ lamellae can, however, also exert an indirect influence on $\lambda$ through a modification of the nucleation behaviour by space or grain boundary occupation (extinction of nucleation sites) or by a modification of the nucleation driving force due to aluminium depletion in $\alpha$.

At high cooling rates, lamellae have only limited time to thicken and the $\alpha$ matrix is not much depleted in Al. Consequently, the driving force for the nucleation of lamellae and the area of grain boundary available for nucleation remain high. This explains why the lamellar spacing is a decreasing function of $\dot{T}$. According to the calculations, at very large cooling rates $\left(\dot{T}>60^{\circ} \mathrm{C} / \mathrm{s}\right), \lambda$ becomes an increasing function of $\dot{T}$, which is due to the lack of time available for nucleation. This decrease is probably not realistic and unlikely to be observed experimentally, as massive $\gamma$ forms at such cooling rates and other nucleation mechanisms may prevail. In order to identify the role of Al depletion in the $\lambda(\dot{T})$ dependency, a simulation was carried out without considering the influence of the matrix depletion on the nucleation rate. The result, which is also shown in Fig. 8, indicates that $\lambda$ is in this case a monotonous increasing function of $\dot{T}$. This result simply translates the fact that the total number of lamellae is obtained by time integration of a continuous nucleation law. It indicates that Al depletion in $\alpha$ during $\gamma$ precipitation plays certainly an important role in the $\lambda(\dot{T})$ dependency.

\subsection{Competition between lamellar and massive $\gamma$}

As can be seen in the CCT diagrams of Fig. 5, the start temperature of the lamellar transformation decreases at high cooling rates, i.e. close to the massive regime. In order to distinguish the respective roles of nucleation and growth in this phenomenon, the number of lamellae per unit length and the final thickness, $w$, have been represented in Fig. 9 as a function of $\dot{T}$. It is observed that the total number of nuclei, $n_{\gamma_{L}}$, increases with $\dot{T}$ in the lamellar regime before diminishing at $\dot{T}>60^{\circ} \mathrm{C} / \mathrm{s}$, consistently with the mechanisms already discussed for the $\lambda(\dot{T})$ dependency. When $n_{\gamma_{\mathrm{L}}}$ starts decreasing, the overall kinetics of the lamellar transformation drops, and the transition from lamellar to massive $\gamma$ becomes possible below $T_{0}$.

The model takes into account three mechanisms that can lead to the suppression of the massive transformation when $T_{0}$ is reached: the space already occupied by the lamellae, the coverage of massive nucleation sites at grain boundaries, and the depletion of the remaining $\alpha$ phase due to lamellar precipitation. The latter effect is expected to be of particular importance, as the driving pressure of the massive transformation decreases very rapidly when the Al content of the $\alpha$ matrix diminishes [35].

Calculations with and without considering the effect of grain boundary coverage ( $s_{\alpha}$ set to either $s_{\alpha}^{\text {out }}$ or 1 in Eq. (1)) have been carried out as an attempt to isolate this effect. The comparison showed only small differences in terms of critical cooling rate for the formation of massive $\gamma$. It can be concluded that, according to the simulations, the coverage of the nucleation sites by lamellae is probably not the dominant mechanism for the suppression of the massive transformation.

The respective role of space occupation by lamellae and $\mathrm{Al}$ depletion in $\alpha$ is more difficult to analyze as they are closely related. The final average concentration in $\alpha\left(\right.$ or $\alpha_{2}$ ) after cooling, $X_{\alpha}^{\text {col }}$, is shown in Fig. 9 as a function of $\dot{T}$. It shows that $X_{\alpha}^{\text {col }}$ is as small as 0.37 at low cooling rates, which is consistent with a transformation taking place close to thermodynamic equilibrium. Massive $\gamma$ can obviously not form in this case as the microstructure is in equilibrium when $T_{0}$ is reached. As already pointed out by Lefebvre et al. [36], the theoretical limit for the $\alpha \rightarrow \gamma_{M}$ transformation is given by $X_{\alpha}^{\text {col }}>X_{\gamma_{M}}^{\min }$, where $X_{\gamma_{M}}^{\min }$ is defined by the intersection of the two $T_{0}$ lines of the $\alpha \rightarrow \alpha_{2}$ and $\alpha \rightarrow \gamma_{M}$ reactions (see Fig. 4). If the Al content in $\alpha$ is sufficiently diminished by the growth of $\gamma$ lamellae to reach $X_{\gamma_{M}}^{\min }$, the massive transformation is likely to be suppressed by the $\alpha \rightarrow \alpha_{2}$ ordering reaction. This condition is verified in Fig. 9 where
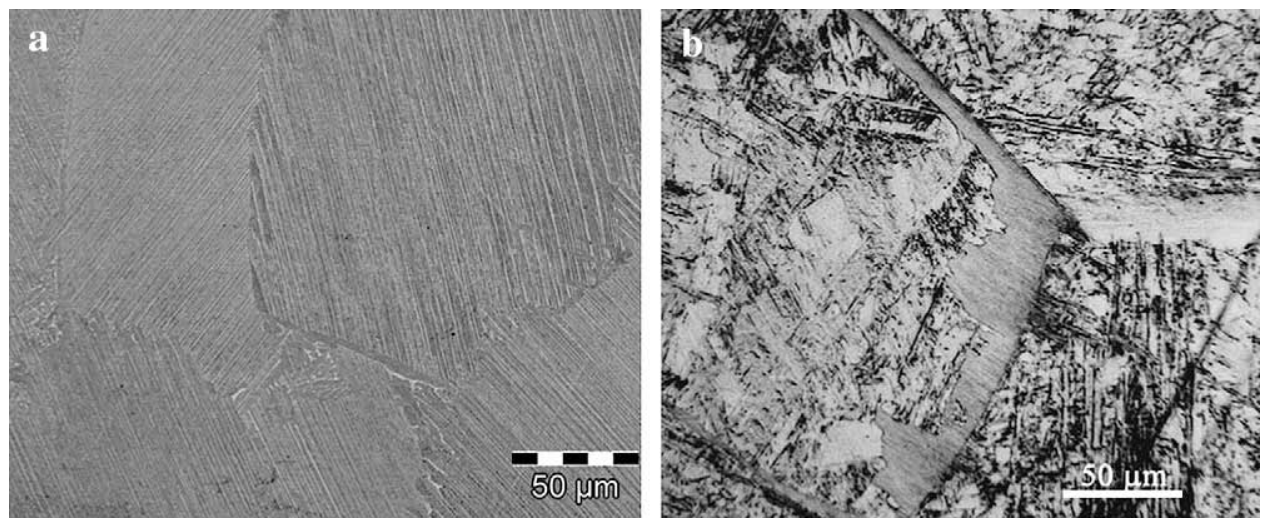

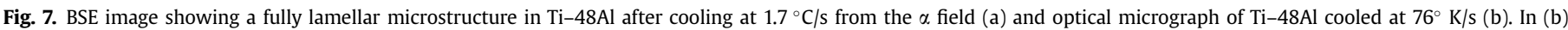
lamellar colonies are observed near the grain boundaries while massive $\gamma$ occupies the interior of the grains. 


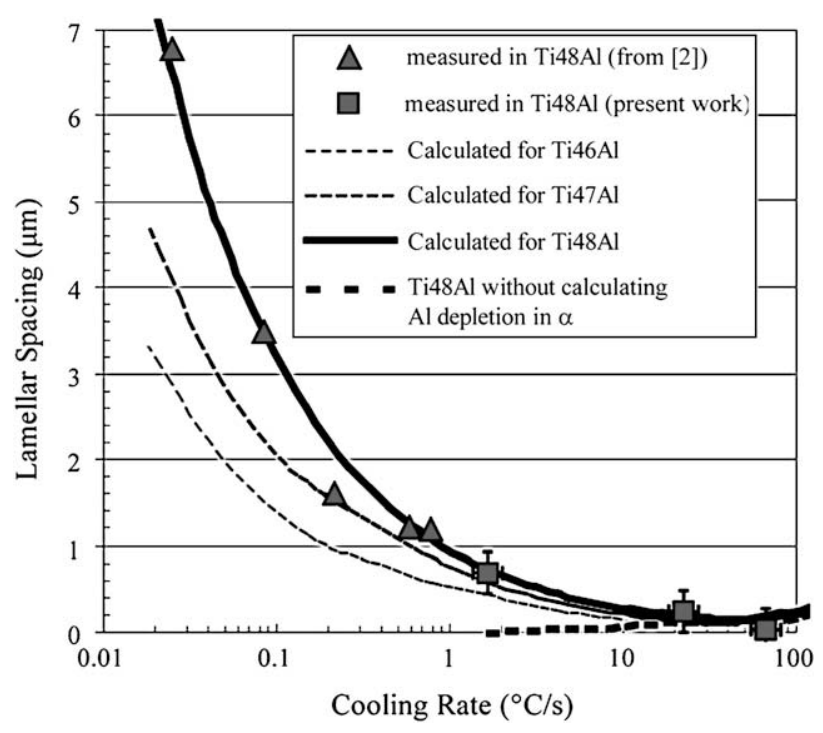

Fig. 8. Lamellar spacing as a function of the cooling rate measured for Ti-48Al and calculated for Ti-46Al, Ti-47Al and Ti-48Al.

the massive microstructure is observed only for $X_{\alpha}^{\text {col }}>0.45$. This concentration is about 0.01 larger than $X_{\gamma_{M}}^{\min }$ due to the undercooling that is necessary to nucleate the massive phase (about $120^{\circ} \mathrm{C}$ ).

The Al depletion in untransformed $\alpha$ predicted by the model depends on the assumption made for the lengthening velocity of the colonies, i.e. on the choice of parameter $b$ in Eq. (14). At low $b$ the colony expansion is fast as compared to the thickening rate and the solute depletion is distributed over a large volume of $\alpha$. In the opposite situation of high $b$ and slow expansion, solute depletion is concentrated on a small volume of $\alpha$ and thermodynamic equilibrium is quickly reached within the colony. At large lengthening rates the influence of $b$ on the proportion of massive and lamellar $\gamma$ tends to saturate. This is due to the fact that the volume fraction of lamellar $\gamma$ is in any case low during the expansion of the colonies. The concentrations of $\alpha$ inside and outside of the colonies are therefore almost identical and massive $\gamma$ can form indistinctly inside or outside the colonies. In the calculations presented here relatively fast lengthening kinetics were adopted $(b=1)$. This can be seen in Fig. 6 where the amount of lamellar $\gamma, g_{\gamma_{L}}$, is rather low when $g^{\mathrm{col}}(\dot{T})$

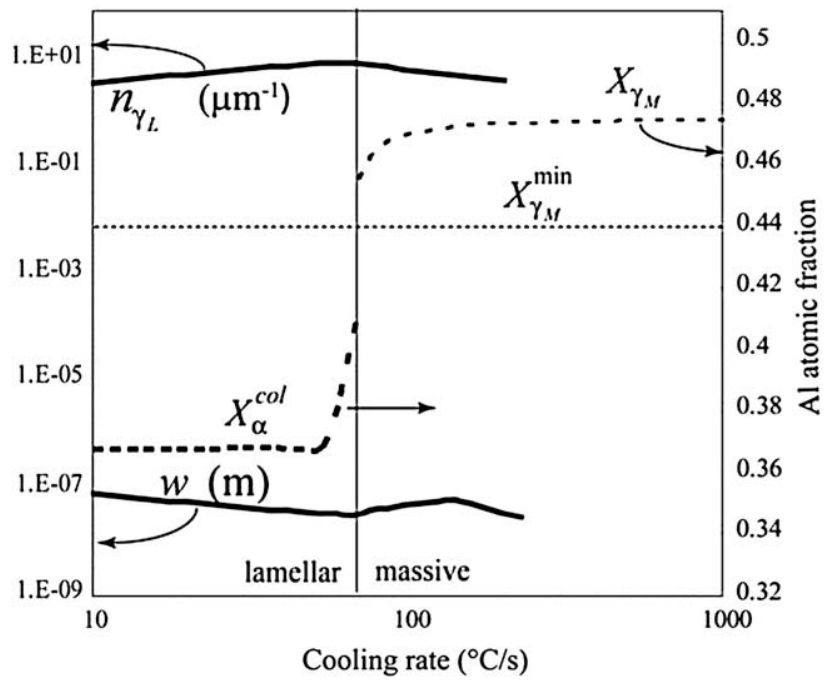

Fig. 9. Calculated final average thickness of the lamellae, $w$, number of lamellae per unit length, $n_{\gamma_{L}}$, and composition of massive $\gamma$ and $\alpha_{2}$ as a function of the cooling rate. starts to drop. With such a choice, the sensitivity of $g_{\gamma_{M}}$ and $g_{\gamma_{L}}$ with respect to $b$ is very low, and the distinction between massive $\gamma$ formed inside and outside the colonies becomes somewhat artificial. Dedicated experiments such as quenching the microstructure at different stages would be needed to better evaluate the colony expansion rate and make this distinction less arbitrary. On the other hand other morphologies such as Widmanstätten and feathery $\gamma$ are likely to form in the transition regime. These morphologies are not included in the presented model.

\subsection{Influence of alloy composition and $\alpha$ grain size}

The model has been used to study the effect of the alloy composition and grain size on the CCT diagram. Fig. 10 shows the predicted CCT diagram for Ti-46Al. As can be seen, the massive volume fraction can hardly exceed $30 \%$ for this composition. The reason is that the difference between the $T_{0}$ temperatures for the $\alpha \rightarrow \gamma_{M}$ and $\alpha \rightarrow \alpha_{2}$ reactions is only $150^{\circ} \mathrm{C}$ for this composition, or in other words, the nominal composition is close to the theoretical limit, $X_{\gamma_{M}}^{\min }$, mentioned previously.

As nucleation of both lamellar and massive $\gamma$ take place predominantly at grain boundaries, the $\alpha$ grain size is potentially an important influencing factor for the kinetics of the phase transformations. The effect on the critical cooling rate for the transition from lamellar to massive $\gamma$ is, however, not obvious since both morphologies develop from the grain boundaries. Fig. 11 shows a calculated CCT diagram for Ti-47.5Al using a grain size of $\bar{R}_{\text {grain }}=400 \mu \mathrm{m}$. By comparing cautiously with Fig. 5b, which was obtained with $\bar{R}_{\text {grain }}=150 \mu \mathrm{m}$, one can observe that a larger grain size slightly depletes the calculated starting temperature of both the lamellar and massive transformations. Also, the critical cooling rate obtained with the model is slightly lower for $\bar{R}_{\text {grain }}=400 \mu \mathrm{m}$ than for $150 \mu \mathrm{m}$. According to the simulations, the influence of the $\alpha$ grain size remains, however, small in comparison with the effect of the composition.

A lower $\mathrm{Al}$ content of the alloy is expected to lead to smaller lamellar spacings for identical cooling conditions. This tendency is predicted with the model as can be seen in Fig. 8 where the calculated spacing in Ti-46Al can be compared with those of $\mathrm{Ti}-47 \mathrm{Al}$ and $\mathrm{Ti}-48 \mathrm{Al}$. The influence of composition on $\lambda$ is explained by the fact that the temperature of transformation increases with the $\mathrm{Al}$ content. At high temperature growth is substantially faster as

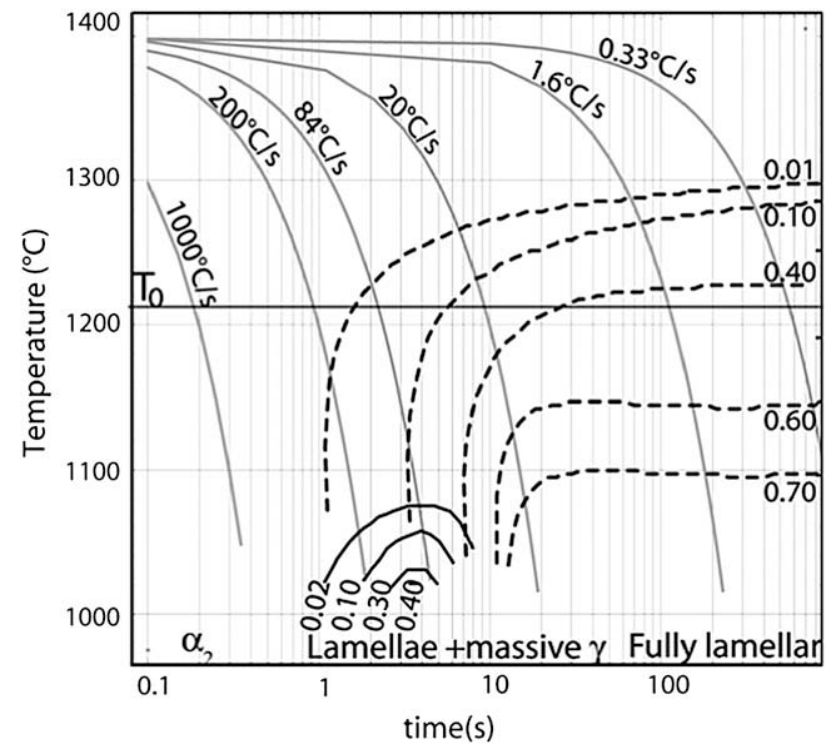

Fig. 10. Calculated CCT diagram for Ti-46Al and an $\alpha$ grain size $\bar{R}_{\text {grain }}=150 \mu \mathrm{m}$. 


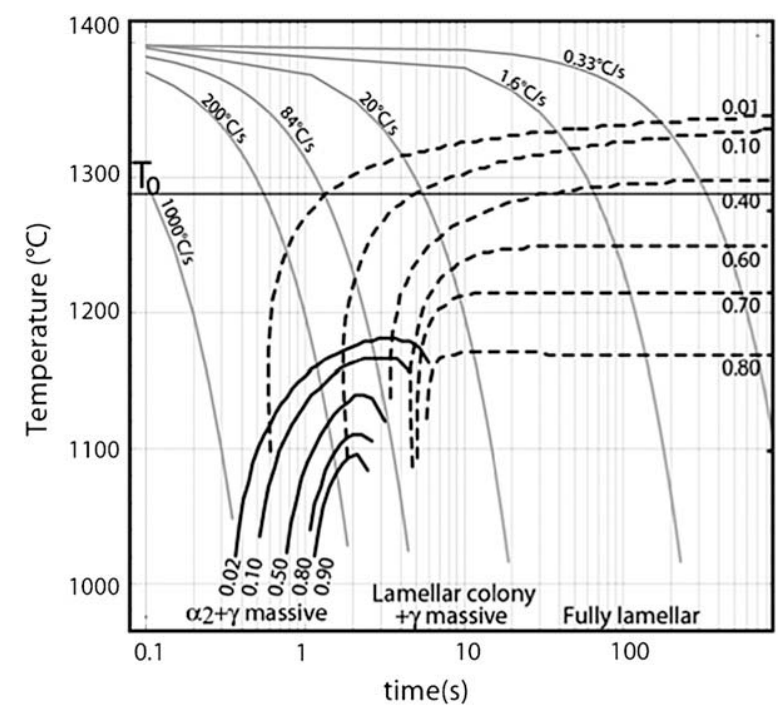

Fig. 11. Calculated CCT diagram for Ti-47.5Al and an $\alpha$ grain size $\bar{R}_{\text {grain }}=400 \mu \mathrm{m}$.

it is a thermally activated process. Faster nucleation rates are also expected at high temperature but this effect is relatively limited due to the rapid decrease of the nucleation driving pressure as growth proceeds.

\section{Conclusion}

The modelling approach which has been developed for the lamellar and massive transformations incorporates the important mechanisms of these transformations, such as nucleation on grain boundary, formation of lamellar colonies, influence of $\alpha$ concentration on the driving pressures. The model can describe the transition from lamellar to massive microstructures in $\gamma$-TiAl as the cooling rate increases. Comparisons with experimental data of transformation temperatures, lamellar spacing and phase proportions showed good agreement. The model was exploited to investigate the mechanisms of competition between nucleation and growth and between lamellar and massive $\gamma$. The main outcome of this study can be summarized as follows:

- At high cooling rate, the $\gamma$ lamellae have only limited time to nucleate and grow before the $T_{0}$ temperature of the $\alpha \rightarrow \gamma$ massive transformation is reached. The massive microstructure is therefore promoted by three factors: (i) the amount of untransformed $\alpha$ is still high at $T_{0}$, (ii) the concentration of untransformed $\alpha$ is still close to the nominal value, (iii) many nucleation sites are still available at grain boundaries. According to the simulations, the third factor seems to have only a limited influence.

- The aluminium depletion in untransformed $\alpha$ associated with the lamellar precipitation can drastically reduce the driving pressure for the massive transformation. The massive transformation is suppressed when the concentration in $\alpha$ becomes close to the theoretical limit given by the intersection of the $T_{0}$ lines of the $\alpha \rightarrow \alpha_{2}$ and $\alpha \rightarrow \gamma_{M}$ reactions.

- A high aluminium content of the alloy tends to favour the massive transformation. However, according to the simulations, the critical cooling rate to obtain massive $\gamma$ seems almost independent of the nominal composition in binary alloys.

- A high aluminium content of the alloy tends to lead to larger lamellar spacings. This effect is associated with the temperature of transformation.
- A smaller grain size accelerates both the lamellar and the massive transformations. According to the simulations, the grain size has almost no effect on the critical cooling rate to obtain massive $\gamma$, as both microstructures develop faster.

In the model a distinction is introduced between massive growing outside and inside the lamellar colonies, which are considered in this approach as the $\alpha$ regions that are depleted in $\mathrm{Al}$. Currently this distinction is somehow arbitrary as data on the expansion rate of the lamellar colonies are missing. Dedicated experiments would be needed to better exploit this aspect of the model. Currently the model is limited to binary alloys. Extension to multi-component systems is underway. This is an important step towards the application of the model to industrially relevant TiAl alloys and towards incorporation into casting and heat treatment simulation packages.

\section{Acknowledgements}

The authors would like to thank Prof. J. Beddoes, from Carleton University (Canada) for having kindly provided the Ti-48Al samples and for very interesting discussions. This work is financially supported by European Commission (contract number NMPCT-2004-500635) 6th Framework Program as the project "Intermetallic Materials Processing in Relation to Earth and Space Solidification", which is co-funded and coordinated by the European Space Agency, with additional project funding contributions from the Government of Switzerland.

\section{References}

[1] Kim YW, Dimiduk DM. JOM 1991:43:40.

[2] Appel F, Wagner R. Mater Sci Eng 1998;22:187.

[3] Chan KS, Kim YW. Acta Metall Mater 1995;43:439.

[4] Wang P, Viswanathan GB, Vasudevan VK. Metall Mater Trans 1992;23A:690.

[5] Zhang WJ, Francesconi L, Evangelista E, Chen GL. Scr Mater 1997;37:627.

[6] Dey SR, Hazotte A, Bouzy E, Naka S. Acta Mater 2005;53:3783.

[7] Zhang WJ, Chen GL, Evangelista E. Metall Mater Trans A 1999;30A:2591.

[8] $\mathrm{Hu} \mathrm{D}, \mathrm{Wu} \mathrm{X}$, Loretto MH. Intermetallics 2005;13:914.

[9] Prasad U, Chaturvedi MC. Metall Mater Trans A 2003;34:2053.

[10] Hu D, Huang AJ, Wu X. Intermetallics 2007;15:327.

[11] Aaronson HI. Metall Mater Trans A 2002;33:2285.

[12] Veeraraghavan D, Wang P, Vasudevan VK. Acta Mater 2003;51:1721.

[13] Veeraraghavan D, Wang P, Vasudevan VK. Acta Mater 1999;47:3313.

[14] Wen YH, Chen LQ, Hazzledine PM, Wang Y. Acta Mater 2001;49:2341.

[15] Katzarov I, Malinov S, Sha W. Acta Mater 2006;54:453.

[16] Jones SA, Kaufman MJ. Acta Metall Mater 1993;41:387.

[17] Denquin A, Naka S. Acta Mater 1996;44:343.

[18] Hono K, Abe E, Kumagai T, Harada H. Scr Mater 1996;35:495.

[19] Dey SR, Bouzy E, Hazotte A. Scr Mater 2007;57:365.

[20] Zhang XD, Godfrey S, Weaver M, Strangwood M, Threadgill P, Kaufman MJ, et al. Acta Mater 1996;44:3723.

[21] Wang P, Veeraraghavan D, Kumar M, Vasudevan VK. Metall Mater Trans A 2002;33:2353.

[22] Dey SR, Bouzy E, Hazotte A. Intermetallics 2006;14:444.

[23] Hu D, Huang AJ, Novovic D, Wu X. Intermetallics 2006;14:818.

[24] Thermo-Calc version P. Stockholm: Foundation for Computational Thermodynamics 2002.

[25] Burke JE, Turnbull D. Prog Metal Phys 1952;3:220.

[26] Hunt JD. Mater Sci Eng 1984;65:75.

[27] Zener C. J Appl Phys 1949;20:950.

[28] Zhao L, Tangri K. Philos Mag 1992;65:1065.

[29] Hsiung LM, Schwartz AJ, Nieh TG. Intermetallics 2004;12:727.

[30] Avrami M. J Chem Phys 1940;8:212.

[31] Dudzinski D, Zhao L, Beddoes J, Wallace W. Scr Mater 1996;35:367.

[32] Recina V, Ahlstrom J, Karlsson B. Mater Charact 1997;38:287.

[33] Köppers M, Herzing CHR, Friesel M, Mishin Y. Acta Mater 1997;45:4181.

[34] Perdrix F, Trichet MF, Bonnentien JL, Cornet M, Bigot J. Intermetallics 1999; 7:1323.

[35] Jacot A, Rostamian A. Mater Sci Forum 2007;539:1481.

[36] Lefebvre W, Loiseau A, Menand A. Metall Mater Trans A 2003;34:2067. 